\title{
WAVELET AND FOURIER DECOMPOSITION BASED PERIODIC NOISE REMOVAL IN MICROSCOPY IMAGES
}

\author{
M.G. IONITA ${ }^{1}$, H.G. COANDA ${ }^{2}$ \\ ${ }^{1}$ Electrical Engineering Electronics and Information Technology, Institute of Multidisciplinary Research for Science \\ and Technology Valahia University of Targoviste Targoviste, Romania, ${ }^{2}$ Electrical Engineering Electronics and \\ Information Technology, Valahia University of Targoviste, Targoviste, Romania \\ Email: marius.ionita@valahia.ro, henri.coanda@valahia.ro
}

\begin{abstract}
The microscopy images can be affected by periodic noise, resulting in quality degradation and the appearance of repetitive patterns on the micrograph. In order to effectively remove the periodic noise, a new adaptive method is proposed in this paper. The presented approach analyzes the frequency domain of the first order Haar Wavelet transform, determines the frequency regions that correspond to noise and corrects the magnitude spectrum components of the undecomposed DFT transform of the image. Experimental results are provided.
\end{abstract}

Keywords: periodic/quasi-periodic noise, frequency domain filtering, Discrete Fourier Transform, Haar wavelet.

\section{INTRODUCTION}

Inaccurate results are obtained if the microscopy images are affected by periodic or quasi-periodic noise [1]. In spatial domain, this type of noise has an undesirable appearance that creates repetitive pattern in different image regions while in the frequency domain it presents high magnitude spike-like spectral components [2]. The denoising implies identification, removal or correction of the peaks related to the noise.

A pure single frequency periodic noise with almost zero bandwidth will create a single peak in the image spectra [6] which can be easily detected and corrected. These cases are rare. Most of the periodic noise is not pure and is formed from multiple frequencies that compose a quasiperiodic structure which can harder be detected and removed.

A few methods that deals with the elimination of periodic noise from microscopy images have already been proposed. For removing the noise components, these methods use Wiener filtering, Gaussian-Notch filters [1], [2], [5], [8], [10], [12], masking filters [7], [9], [11], [13], spectral component value replacement based on spectrum statistics [3], [4], [13], etc.

A method with good results has been published in [12]. The basic idea of [12] is the detection of noise components in the Fourier domain by using an average power spectra computed from a set of patches of the noisy image. In order to determine the noise related peaks, a power law distribution is fitted to the computed spectrum using a robust linear regression algorithm. The high value outliers corresponds to the noise peaks. A map with the positions of the outliers in the Fourier domain is computed and furthermore, a notch filter is used to eliminate the noise components.

In [15], recently, has been proposed a frequency domain filter that incorporates a adaptive threshold based noise detection scheme. The detection method is used to effectively approximate the uncorrupted version of frequency domain surface to differentiate corrupted frequencies which are further replaced with the minimum of uncorrupted spectra components.

In our previous work, [16], we proposed an automated method that analyzes the frequency domain to detect potential noise components in order to remove them. More precisely, the amplitude spectrum is processed with two filters and an iterative thresholding method in order to detect the noise components. Two masks are designed for the detected components. These masks are used to process the spectrum in order to eliminate the periodic noise.

In this paper we propose an improved method for detecting and removing of periodic and quasi-periodic noise patterns from microscopy images. More precisely, the image is decomposed with 2-D discrete wavelet transform in four bands, each sub-band is processed using the method from [16] and a first filtered image is obtained by recomposing it from the filtered DWT bands. The frequency spectra of the initial image and the one after the first step are used to design two masks for the elimination of periodic noise components. Since the methods proposed in [12], [15], [16] have good results, they will be used for comparisons.

The structure of the paper is as follows. The proposed method is described in Section II. Experimental results are presented in Section III. Finally, the conclusions are drawn in Section IV.

\section{PROPOSED METHOD}

The periodic and quasi-periodic noise patterns are very difficult to remove without affecting the image contours and fine details. The use of frequency domain for image denoising improves the quality of results, but it depends on a very accurate identification of noise components from the spectrum.

The proposed method utilizes the first level Discrete Wavelet Transform for splitting the information into four 

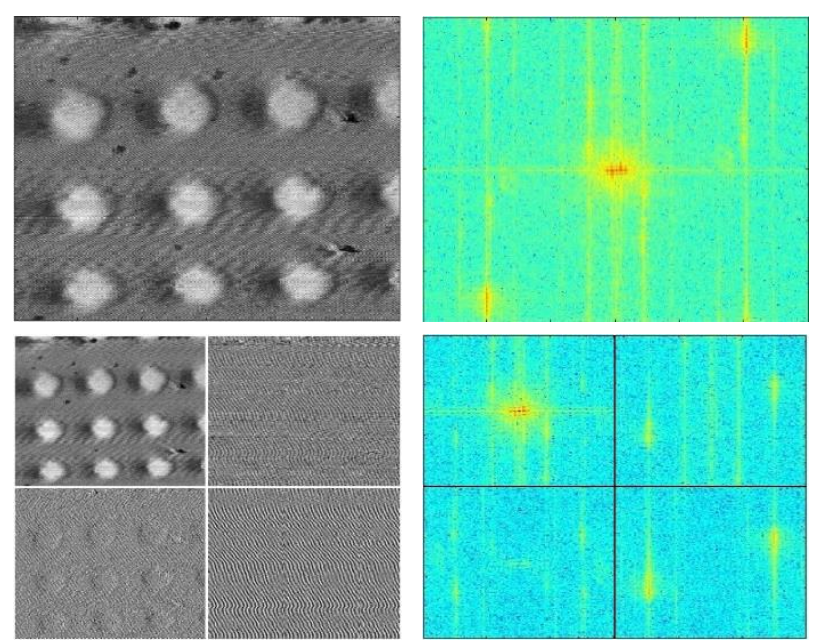

Figure 1. Wavelet decomposition: Original image and its spectrum (top row); Wavelet decomposition and its spectras (bottom row)

sub-bands (Figure 1), namely image approximation, horizontal, vertical and diagonal details. This decomposition basically facilitates the detection of specific noise components that are usually hidden by the spectrum general shape or by high magnitude neighbor frequency components.

Each DWT sub-band is filtered using the method presented in [16]. The magnitude spectrum $M$ is filtered with a sliding window equal with a quarter of minimum value between the height and width of the image. The spectrum shape is normalized and a first noise detection using the next equations.

$$
\begin{gathered}
M n_{i, j}^{\prime}=\frac{M_{i, j}}{Q_{i, j}}-1 \\
\left(M n_{0}^{\prime}\right)_{i, j}=\left\{\begin{array}{cc}
0 & \text { if } M n_{i, j}^{\prime}<0, \\
0 & \text { if } M n_{i, j}^{\prime} \in \text { protected area }, \\
M n_{i, j}^{\prime} & \text { otherwise }
\end{array}\right.
\end{gathered}
$$

where $M_{i, j}$ is the current spectrum component for which the normalization is computed and $Q_{i, j}$ is the average of the elements around $M_{i, j}$ in the window.

An iterative enhancement is performed on the resulted spectrum as described by the following equations.

$$
\begin{gathered}
Q_{i, j}^{\prime}=\frac{\left(\sum_{k=-1}^{1} \sum_{p=-1}^{1}\left(M n_{0}^{\prime}\right)_{i-k, j-p}\right)-\left(M n_{0}^{\prime}\right)_{i, j}}{8} \\
M e_{i, j}=\log \left(1+\left(M n_{0}^{\prime}\right)_{i, j} Q_{i, j}^{\prime}\right)
\end{gathered}
$$

The next step is the thresholding of the enhanced spectrum. The threshold $\mathrm{T}$ is computed using the algorithm presented in [14].

$$
N_{c_{i, j}}=\left\{\begin{array}{cc}
0 & \text { if } M e_{i, j}<T \\
M e_{i, j}-T \text { otherwise }
\end{array}\right.
$$
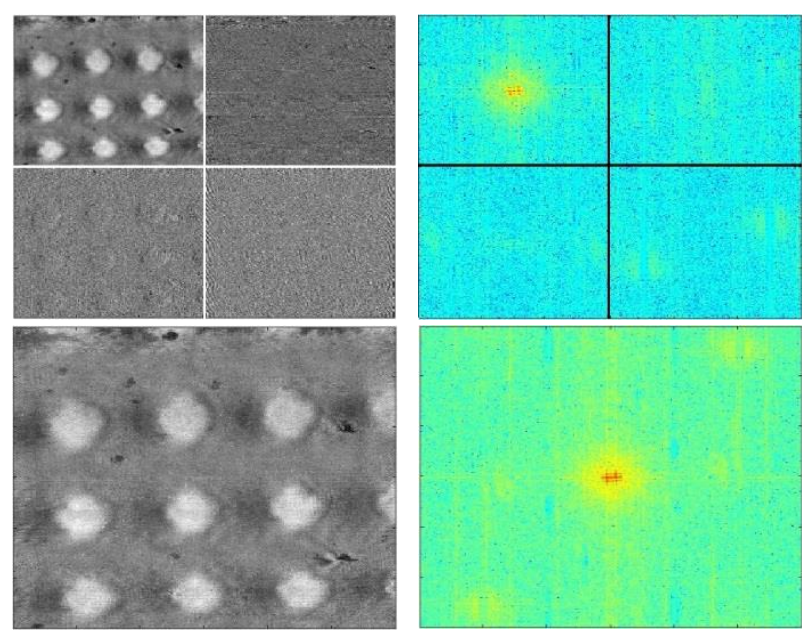

Figure 2. Filtering the wavelet sub-bands: Filtered subbands and the spectras (top row); Image after recompose and its spectra (bottom row).

The noise components map $N_{c}$ is used to create two correction masks which are applied over the initial spectrum to eliminate the periodic noise. The filtered subband is obtained by computing the Inverse Fourier Transform of the corrected spectrum (Figure 2).

After the filtration of each sub-band the image is recomposed by applying the Inverse Wavelet Transform. As it can be seen in Figure 2 we obtained a filtered image. The micrograph is not noise free, a few noise components of small amplitudes can still be observed in the frequency domain $M_{w}$, but it can be used to obtain a map $M_{f}$ of the noise components from the original spectrum $M$.

$$
\begin{aligned}
& M_{f_{i, j}}=\left|M_{i, j}-M_{w_{i, j}}\right| \\
& M_{f_{i, j}}=\left\{\begin{array}{cc}
0 & \text { if } M_{f_{i, j}<0.1} \\
M_{f_{i, j}} & \text { otherwise }
\end{array}\right.
\end{aligned}
$$

The amplitudes smaller than 0.1 are not taken into consideration because they usually are leftovers from the recomposition. Also, special care has been taken for avoiding the introduction of unwanted artefacts by affecting the low frequency area. This area is supposed to have a circular shape and its radius is computed by using our method described in [13].

The resulted spectrum can be observed in Figure 3. a). A first amplitude reduction mask, $M_{r}$, is created by convolving the Gaussian window $G$ with the noise component spectrum $M_{f}$.

$$
\begin{gathered}
G_{x, y}=\frac{1}{2 \pi \sigma^{2}} e^{-\frac{x^{2}+y^{2}}{2 \sigma^{2}}} \\
M_{r}=1-\left(\frac{G * M_{f}}{\arg \max \left(G * M_{f}\right)}\right)
\end{gathered}
$$


where the window size of $G$ is equal to the radius of the central region of $M_{w}$ spectrum and $\sigma$ is 2.5. The Figure 3, b) shows the effect of applying the mask $G$ over the initial spectrum. Based on the noise map $M_{f}$ the corresponding components of the initial image amplitude spectrum are replaced by the average of nonzero magnitude values found at the same distance from the centre of spectrum (Figure 3, c). The inverse Fourier Transform is computed and the filtered image is obtained (Figure 3,d).

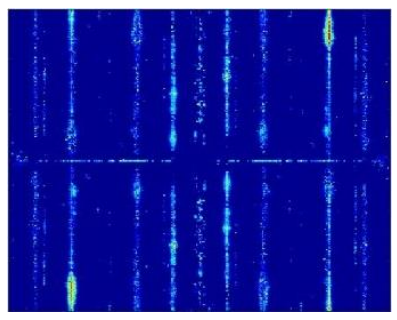

a)

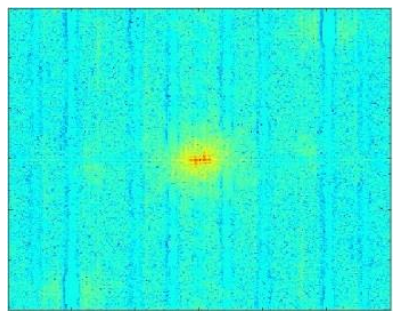

c)

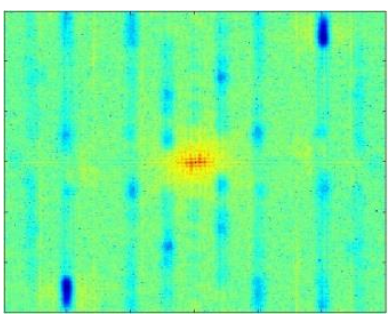

b)

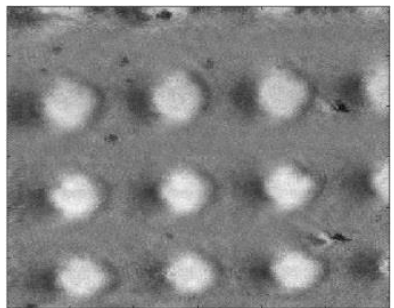

d)
Figure 3. Final filtration operations: a) Noise component map; b) Spectrum after the first mask; c) Filtered magnitude spectra; d) Filtered image.

\section{EXPERIMENTAL RESULTS}

The first experiment uses a reference image with multiple noise components progressively added to evaluate the filtering of multiple noise patterns. For comparison, the methods proposed by F. Sur and M. Grédiac [12] (ARPENOS), J. Varghese [15] (Varghese Filter) and our previous method [16] (FTT9) are used for the same images. To compare the results the Peak-to-peak Signal to Noise Ratio (PSNR) is used.

The results shown in Figure 4 for one up to 50 noise added components prove that while all the methods perform well in removing the simulated noise, the proposed method has slightly better overall results.

The second experiment involves the denoising of real microscopic images affected by periodic noise. As it can be seen in Figure 5, the proposed method performs well in removing the periodic noise from the microscopic images. In terms of better removing the noise components and keeping the fine details like contours, shadows and other definitory aspects of the objects intact, the proposed method outperforms the others.

\section{CONCLUSION}

An improved method for filtering the periodic noise patterns in microscopy images has been proposed. The method uses the Wavelet transform to improve the automatic detection and removal of the components that corresponds to periodic and quasi-periodic noise in the spectral domain. The results obtained so far are very promising.
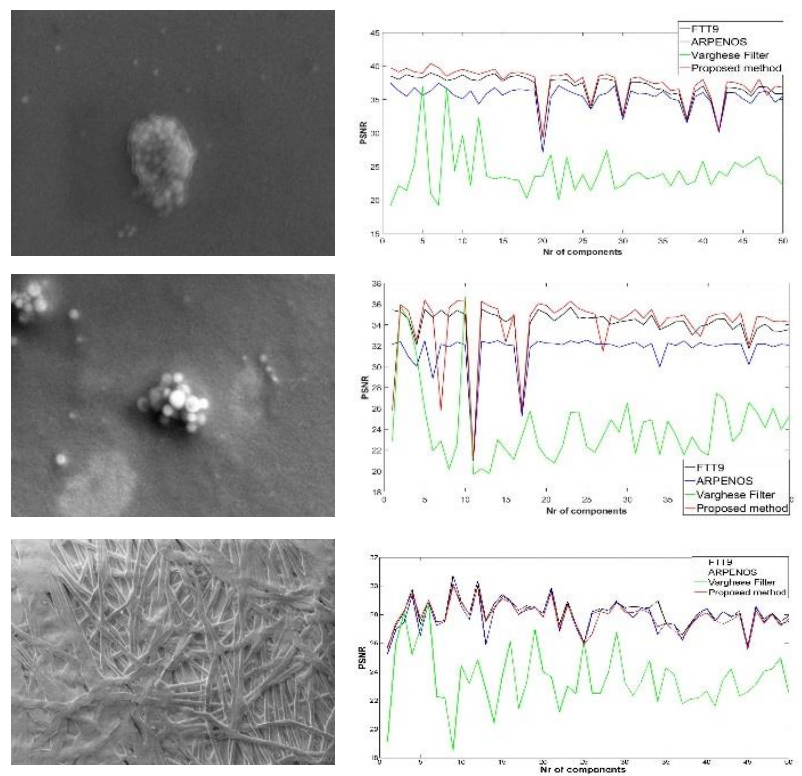

Figure 4. Experimental results of filtering for generated noise with 1-50 noise components: Test Images (left); Experimental results (right).

\section{ACKNOWLEDGEMENT}

The authors thank Dr. Ioana Dulama and Dr. Sofia Teodorescu for providing the microscopic images of Figure 4 and Dr. Stefan Stanciu for the Scanning Nearfield Optical Microscopy (SNOM) images of Figure 5. 

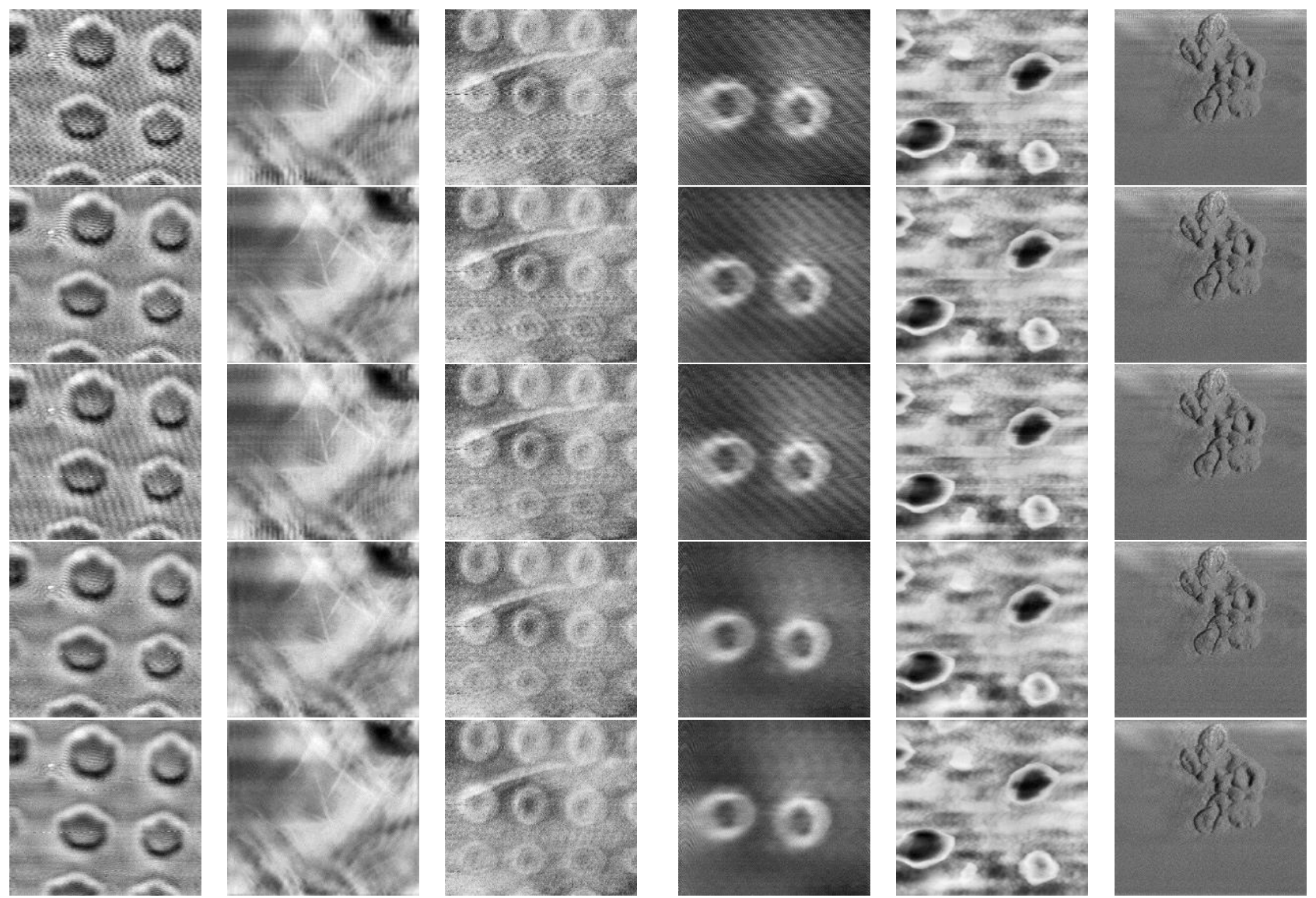

Figure 5. Experimental results of filtering real micrographs: Original images (first row); Denoised images with the method proposed by F. Sur and M. Grédiac (second row); Denoised images with the method proposed by J. Varghese (third row); Results of our previous method [16] (fourth row); Results of the proposed method (fifth row).

\section{REFERENCES}

[1] Z. Wei, J. Wang, H. Nichol, S. Wiebe and D. Chapman, "A median Gaussian filtering framework for Moiré pattern noise removal from X-ray' microscopy image", Micron., vol. 43, no. 2, pp. 170176.

[2] R. Gonzalez, R. Woods, “Digital Image Processing”, 2nd ed. PrenticeHall., Englewood Cliffs, NJ. 2001.

[3] N. Sidorov and Anil C. Kokaram, "Suppression of moiré patterns via' spectral analysis", 2011.

[4] I. Aizenberg, C. Butakoff, "Frequency domain median-like filter for periodic and quasi-periodic noise removal", In SPIE Proceedings of Image Processing: Algorithms and Systems., vol. 4767, pp. 181191., 2002.

[5] I. Aizenberg, C. Butakoff, "A windowed Gaussian notch filter for quasiperiodic noise removal", Image and Vision Computing., vol. 26, pp. 13471353, 2008.

[6] L. Yaroslavsky, M. Eden, Fundamentals of digital optics Birkhauser, Boston, 1996.

[7] R. Arthur, J. Weeks, "Fundamentals of Electronic Image Processing”, IEEE Press., New York, 1996.

[8] J.C. Russ, "Forensic Uses of Digital Imaging", CRC., Boca Raton, FL, 2001.

[9] L. Yaroslavsky, M. Eden, "Fundamentals of Digital Optics", Birkhauser, Boston, 1996.
[10] V. Govindaraj, S.Saravanakumar, V. Gokul durai, "Removal of Moiré Pattern Noise in Images using Median and Gaussian Filter", International Journal of Science, Engineering and Technology Research, vol. 2, no. 2, February 2013.

[11] S. Chen, J. Pellequer, "DeStripe: frequency-based algorithm for removing stripe noises from AFM images”, BMC Structural Biology, 2011.

[12] F. Sur, M. Grediac, "Automated removal of quasiperiodic noise through' frequency domain statistics", SPIE Journal of Electronic Imaging, vol. 24, no. 1, pages 013003/1-19, February 2015.

[13] G. Ionita, D. Coltuc,S. Stanciu, D. Tranca, "Automatic moire pattern' removal in microscopic images", 19th International Conference on System Theory, Control and Computing, pp. 776 - 779, Cheile Gradistei, Romania, October 2015.

[14] T.W. Ridler, S. Calvard, "Picture thresholding using an iterative selection method", IEEE Transactions on Systems, Man, and Cybernetics 8:630632,1978.

[15] Justin Varghese, "Adaptive threshold based frequency domain filter for periodic noise reduction", International Journal of Electronics and Communications (AE) 70, 16921701, 2016.

[16] Marius G. Ionita, Henri George Coanda, "Automatic periodic noise removal in microscopy images", International Symposium on Signals Circuits and Systems - ISSCS 2017, Iasi, Romania, July 2017. 